\title{
Dinámicas urbanas y transición hacia espacios metropolitanos: el caso de Valdivia y la Región de Los Ríos, Chile ${ }^{1}$
}

\author{
Dinâmica urbana e transição para espaços metropolitanos: o caso de Valdivia \\ e a Região de los Ríos, Chile
}

Urban dynamics and transition to metropolitan spaces: the Valdivia case and Ríos Region, Chile

\author{
Francisco Maturana[a] (1D), Fernando Peña-Cortés[b] (D), Francisco Ramírez Carrasco[b] (D), \\ Magdalena Telias[a] (D)
}

[a] Universidad Alberto Hurtado, Departamento de Geografía, Santiago, Región Metropolitana, Chile

[b] Universidad Católica de Temuco, Laboratorio de Planificación Territorial, Temuco, Región de La Araucanía,

Chile

Cómo citar: Maturana, F., Peña-Cortés, F., Ramírez Carrasco, F., \& Telias, M. (2019). Dinámicas urbanas y transición hacia espacios metropolitanos: el caso de Valdivia y la Región de Los Ríos, Chile. urbe. Revista Brasileira de Gestão Urbana, 11, e20180143. https://doi.org/10.1590/2175-3369.011.e20180143

\section{Resumen}

El proceso de urbanización avanza de manera veloz y afecta a múltiples partes del planeta. Así, algunas ciudades de tamaño medio comienzan a evidenciar un proceso de metropolización en sus espacios y en el caso de Chile sus urbes no han estado ajenas a tal proceso. Bajo tal contexto, este artículo buscó comprender las dinámicas de crecimiento en población, variación de superficie construida, verticalización y variaciones del empleo en diferentes áreas económicas de la ciudad de Valdivia, Chile. Se observa un aletargamiento en las tasas de crecimiento de tal ciudad, tanto en su contexto regional como nacional, lo cual se valora de manera positiva al no polarizar su espacio regional. No obstante, existe un incipiente proceso de expansión urbana y crecimiento inmobiliario en altura, vinculado a una tercerización de sus actividades productivas, lo cual es un llamado de atención para los planificadores y el futuro de la sustentabilidad de la urbe, considerando los desafíos que presenta la ciudad en términos de la generación de instrumentos actualizados de planificación territorial, los cuales no están presentes hasta la fecha.

Palabras clave: Valdivia. Ciudades intermedias. Expansión urbana. Verticalización.

\section{Resumo}

O processo de urbanização avança rapidamente e afeta várias partes do planeta. Assim, algumas cidades de porte médio começam a mostrar processos de metropolização em seus espaços. No caso do Chile, suas cidades não desconhecem esse processo. E, nesse contexto, este artigo buscou entender a dinâmica de crescimento populacional, variação de superfície e verticalização e variações de emprego em diferentes áreas econômicas da cidade de Valdivia, Chile. Há uma letargia nas taxas de crescimento de tal cidade,

\footnotetext{
${ }^{1}$ Este artículo se desarrolló bajo el marco del FONDECYT Iniciación № 11150087 y FONDECYT Regular 1181954
}

FM es doctor en Planificación Territorial Urbanismo y Dinámicas del Espacio, e-mail: fmaturana@uahurtado.cl

FPC es doctor en Ciencias Ambientales, e-mail: fpena@uct.cl

FRC es doctor en Gestión y Valoración Urbanística, e-mail: framirez@uctemuco.cl

MT es licenciada en Geografía, e-mail: maga.telias@gmail.com 
tanto em seu contexto regional quanto nacional, que é valorizado de forma positiva por não polarizar seu espaço regional. Além disso, há um incipiente crescimento processo de expansão urbana e imobiliário em altura, ligado à terceirização de suas atividades produtivas, que é um alerta para os planejadores e para o futuro da sustentabilidade da cidade, considerando os desafios apresentados pela cidade em termos de geração de instrumentos atualizados de planejamento territorial, que não estão presentes até hoje.

Palavras-chave: Valdivia. Cidades médias. Expansão urbana. Verticalização.

\section{Abstract}

The process of urbanization advances rapidly and affects multiple portions of the world. In this way, some medium-sized cities have begun to demonstrate metropolisation of their spaces. This article seeks to understand the dynamics of population growth, as well as constructed and functional surface, in different economic areas of employment in the city of Valdivia, Chile. There is a delay in the growth rates of this city, both in a regional and national magnitude. This is considered beneficious given it avoids polarization of its region. Also, there is an incipient real estate growth for an outsourcing of their productive activities, which should be considered as a wake-up call for planners and the future of sustainability.

Keywords: Valdivia. Medium-sized cities. Urban sprawl. Verticalization.

\section{Introducción}

Según cifras de la Organización de las Naciones Unidas (ONU) la población urbana desde 1960 al año 2016 ha aumentado considerablemente en desmedro de la población rural. Para la escena mundial este aumento se sitúa de un 34\% al 54\% (ONU, 2016), pero si orientamos la mirada para el caso de América Latina y el Caribe la brecha de migración urbano-rural se acentúa, pasando de un $49 \%$ a un $80 \%$ respectivamente (ONU, 2016). En este contexto, Montero \& García (2017) en el estudio de la Comisión Económica para América Latina y el Caribe (CEPAL) sobre desarrollo urbano, indican que en América del Sur el 83\% de la población es urbana.

En este proceso de migración y sobre urbanización, las ciudades han generado -a partir de la concentración geográfica y social- un excedente de producción, expresado en procesos de concentración y centralización de capitales (Ramírez, 2014), entendiendo que las políticas de crecimiento de económico requieren espacios rentables para la producción, absorción y recirculación de capital (Harvey, 2013).

En efecto, Santos (2000) plantea que la urbanización estaría condicionada por las estructuras sociales de producción y reproducción de las riquezas, tanto a escala nacional como global, específicamente plasmada en una división territorial del trabajo.

Tal proceso urbanizador y de división del trabajo, estaría intrínsecamente vinculado a la globalización debido a la creciente liberalización económica de los mercados nacionales, su creciente inserción a las redes globales; y las reestructuraciones espaciales que se han originado en las ciudades (Borja, 2005; Castells, 1995; Smith, 2005), las cuales plasman una constante metamorfosis respecto a su estructura espacial permitiendo concentrar flujos y participación en la economía mundial (Borja, 2005). En efecto, tal proceso de capitalismo financiero en áreas urbanas podría situarse a partir de 1990 en las cuales el proceso ha ido acompañado de una creciente gentrificación en los centros de las ciudades (Smith, 1996) en todas partes del mundo, mismo las ciudades latinoamericanas (Mattos, 2010).

Así, esta constante metamorfosis espacial ha sido el resultado de políticas público-privadas que buscan mejorar los indicadores de inversión, productividad y competitividad económica regional. Lo que Schumpeter ha denominado "destrucción creativa" (Montoya, 2012). La destrucción creativa es el tránsito de una estructura de producción antigua a una nueva, y que se materializa sobre el uso del suelo. Los agentes económicos destruyen y reemplazan su capital fijo, medios de producción, infraestructuras físicas y sociales, de acuerdo al ritmo del avance de las nuevas tecnologías, para mantenerse competitivos. En este sentido, Schumpeter plantea que la economía capitalista sería un sistema de producción que se 
encuentra en constante evolución a la par de procesos de innovación (Sapir, 2004). La transformación del proceso, se desencadena cuando usos de suelos establecidos quedan obsoletos en cuanto a su rentabilidad y por tanto se crean nuevas alternativas de rentabilidad (espacios) en la ciudad.

La destrucción creativa no es un fenómeno nuevo, responde a un sistema dinámico de producción en permanente evolución y periódicamente dominado por oleadas de innovación (Montoya, 2012), por tanto, es, en resumen, la creación de nuevas cadenas de producción para mantener niveles competitividad. En este contexto, los bienes raíces y la tenencia de la propiedad pasan de su concepción clásica de mercado del suelo para convertirse en mercancía, extrayendo recursos según las expectativas de rentas futuras (Harvey, 2013). Esto último gatilla la restructuración progresiva de los territorios, impactando no sólo a los espacios metropolitanos, como por ejemplo en procesos de gentrificación, sino que también en las ciudades intermedias (Bellet et al., 2015), las cuales en sus diferentes contextos de sistemas urbanos nacionales se ven enfrentados a nuevos desafíos (Maturana et al., 2017).

En este sentido, gracias a los avances tecnológicos en transportes, telecomunicaciones, nuevas lógicas de movilidad, sistemas productivos y a pesar del tamaño ${ }^{2}$, las ciudades de escala media se han transformado en espacios de suma importancia tanto para sus hinterland como su espacio regional (Maturana \& Rojas, 2015).

América Latina no ha estado ajena a tales procesos de urbanización e impacto de la globalización a diferentes escalas (Borsdorf et al., 2009; Marengo \& Monayar, 2012). En efecto, existió un impulso de la estructura económica urbana en sus funciones y actividades que se orientaron a una producción de orden mundial de los servicios avanzados para empresas (Mattos, 2010), que expresa el crecimiento de los mercados internos de los países de América Latina (Marengo \& Monayar, 2012).

Por ejemplo, el proceso de industrialización que comenzó en ciudades como Sao Paulo y Buenos Aires, abrió paso a la apertura de la economía latinoamericana a una escala global, atrayendo capitales principalmente norteamericanos y británicos que penetraron las economías de la región, potenciando el desarrollo de centros económicos y urbanos, actualmente impactando a otras ciudades en sus respectivos sistemas urbanos nacionales (Bitoun et al., 2017; Lan \& Migueltorena, 2017).

Chile tampoco ha quedado exento a este proceso, ya que se caracteriza por su elevado grado de liberalización económica correspondiente a uno de los más altos de la región. En efecto, la conexión entre los mercados chilenos y economía mundial se encuentra consolidada (alto nivel de exportaciones e inversiones de capital extranjero en territorio chileno), y se expande por todos los ámbitos de producción y servicios, debido a que a la tradicional exportación de cobre que caracteriza el país, se han sumado el ámbito agrícola, pesquero y forestal, incluyendo capitales transnacionales en sectores financieros (Barton et al., 2007).

Bajo tal contexto, se ha desarrollado un importante proceso de urbanización que actualmente supera el 87\% (INE, 2017) y cuya materialización no es sólo posible de apreciar en los tres espacios metropolitanos del país (Santiago, Valparaíso y Concepción), sino que también en un número importante de las denominadas ciudades medias o intermedias, las cuales algunas estarían presentando rasgos de metropolización de sus espacios y por tanto desafíos en términos de planificación urbana (Maturana et al., 2018; Santiago et al., 2016).

Las ciudades medias o intermedias en Chile, comienzan a tener una mayor preocupación por parte la comunidad científica a mediados del año 2000 (Maturana \& Rojas, 2015) ${ }^{3}$, cuando aparecen una serie de textos referentes a problemáticas vinculada a la expansión urbana de las ciudades y el rol que tales urbes generan en sus espacios regionales. Así, se planteó el año 2009 (Hidalgo et al., 2009), la idea del paso de un país urbano a uno metropolitano principalmente en las capitales regionales (incluso a algunas provinciales), considerando los desafíos que esto implica en términos de planificación urbana y contextualizado en la actualidad en el debate de sostenibilidad que presentan las ciudades bajo el modelo económico de mercado en el que se encuentran insertas.

\footnotetext{
${ }^{2}$ Comprendido tanto en su extensión espacial como cantidad de población.

${ }^{3}$ Se refiere a la producción científica de artículos, lo cual no quita la importancia de trabajos que las universidades regionales han podido desarrollar a nivel de pregrado desde hace ya bastante tiempo.
} 
Un caso paradigmático en el sistema urbano chileno, corresponde a la ciudad de Valdivia en la Región de los Ríos. Tal ciudad, hasta mediados del siglo XX, era una de las urbes más importantes del sur de Chile, pero posteriormente evidenciaría un aletargamiento demográfico (Borsdorf, 2000), que de forma positiva se podría visualizar como una posibilidad de potenciarla como ciudad sustentable y con una preocupación mayor por el medioambiente, que pudiese emanar de un ordenamiento territorial (Borsdorf et al., 2009).

Valdivia, si bien fue cabecera provincial (de la Región de Los Lagos), se le otorga el año 2007 el rango de capital de la Región de Los Ríos y es bajo esta perspectiva, que este trabajo buscó explorar, las dinámicas de crecimiento en población y superficie que experimenta la ciudad. Ambos, bajo un contexto de transformaciones funcionales en diferentes áreas económicas del empleo.

Se planteó la hipótesis que existe un aletargamiento en las tasas de crecimiento de tal ciudad tanto en su contexto regional como nacional, no obstante que existe un incipiente, pero relevante, crecimiento del producto inmobiliario en altura vinculado a una tercerización de sus actividades productivas, lo cual es un llamado de atención para los planificadores y el futuro de la sustentabilidad de la urbe.

Para dar respuesta a tal interrogante, se caracterizó en términos de su evolución en población (contexto local y regional), superficie (mediante imágenes satelitales) y crecimiento en altura (un terreno realizado) experimentada en los últimos años. Finalmente, se exploran análisis de empleo en diferentes áreas de la económicas gracias a datos del censo de población para dar cuenta de la evolución y transformación económica de tal espacio de la ciudad en su contexto regional.

\section{Materiales y métodos}

Para la realización de este trabajo se llevaron a cabo cuatro pasos. El primero consistió en analizar la evolución de población de la ciudad en cuestión, tanto en su contexto regional como nacional. Así, se elaboró la tasa de crecimiento desde el año 1952 a 2017 para todas las ciudades ${ }^{4}$ de la Región de Los Ríos. En el contexto nacional, se consideraron los datos desde el año 1930 hasta 2017, en base a la disponibilidad de información. Todos los anteriores fueron extraídos de los documentos del Instituto Nacional de Estadísticas de Chile (INE, 1999, 2005 y 2017), teniendo presente que, en los datos del nuevo censo del año 2017, se extrajo la parte urbana de tales comunas (excluyendo la rural), haciendo así más representativo el dato.

El segundo paso fue estimar la superficie edificada de la ciudad. Se procedió a recopilar y descargar imágenes satelitales Landsat ${ }^{5}$ para el área de estudio en cuatro períodos 1998, 2001, 2011 y 2015. Así, se realizó la vectorización de la superficie construida (considerando el límite urbano censal como referencia para cada uno de los períodos ${ }^{6}$ ). Lo anterior, se generó a través de la delimitación de polígonos correspondientes para cada una de las imágenes antes señaladas a partir de una interpretación visual de las imágenes. Para subsanar errores en la interpretación (por ser visual), la delimitación del polígono fue comparada constantemente con Google Earth a partir de la herramienta de imágenes históricas. En una etapa posterior se realizó a través del software QGIS la medición de la superficie en cada uno de los polígonos delimitados para los años ya indicados, y con el resultado obtenido se realizó el cálculo de variación porcentual (en base a lo propuesto por Aguayo et al., 2009), con el objetivo de observar el porcentaje de crecimiento de la superficie construida de un año a otro.

Un tercer paso fue analizar el proceso de crecimiento en altura o verticalización vinculada a la función residencial-habitacional mediante la información de permisos de edificación por actividad para los años 2002 y 2015 proporcionada por el INE (2016). Tales datos se presentaron en una serie temporal para clarificar el rol de la edificación y otro tipo de construcción en la verticalización de la ciudad. Además, se

\footnotetext{
${ }^{4}$ Entidades sobre los 5.000 habitantes según el Instituto Nacional de Estadísticas de Chile, en el año 2005.

${ }^{5}$ Fueron bajadas desde el sitio USGS (2018).

${ }^{6}$ Para el año 1988 se utilizó el de 1992, para el del 2001 el límite urbano censal del año 2002. Finalmente, para el año 2015 se utilizó el límite urbano censal del 2016.
} 
realizó un trabajo de campo en el área de estudio durante el mes de marzo del 2017, en donde se generó un terreno que permitió reconocer todas las calles de la ciudad de Valdivia, para proceder a una georreferenciación de los edificios de carácter habitacional. No se consideraron edificios en construcción, conjuntos habitacionales tipo block, ni edificaciones comerciales.

Posteriormente, se realizó una sistematización de la información recopilada, para luego utilizar la técnica Densidad de Kernel con el objetivo de analizar espacialmente el patrón de los puntos georreferenciados. Para lo anterior, en una primera instancia se obtuvo el análisis de vecinos más próximos del total de los puntos ingresados. Este análisis calculó la distancia media observada, la distancia media esperada y el índice de vecino más próximo del total de los puntos ingresados. Con estos resultados, se procedió a realizar la Densidad de Kernel en el software QGIS. El resultado de este proceso, se reclasificó en cinco rangos de mayor a menor densidad respectivamente "Muy alto", "Alto", "Medio", "Bajo" y "Muy bajo".

Un último y cuarto paso, fue caracterizar -mediante el empleo- funcionalmente la ciudad. Para tal afecto, se elaboró un gráfico en la evaluación de tal característica para la ciudad de Valdivia entre los años 1982, 1992, 2002 y 2017, todos los anteriores, extraídos de los respectivos censos.

Tal análisis, se complementó con un análisis de componentes principales (ACP) que permitía comprender -considerando el conjunto de centros urbanos de la región- la evolución del empleo en distintas ramas económicas (Pumain \& Saint-Julien, 2001). Para llevar a cabo tal proceso, se utilizaron los mismos años ya indicados.

Es necesario considerar, que tales datos utilizan la Clasificación Internacional Industrial Uniforme de la Organización de las Naciones Unidas (ONU) para caracterizar el sector económico en que trabajan los habitantes y si bien existe una leve variación entre los años 1982-1992-2002 y el 2017 se ajustaron las variables para su análisis. Los análisis anteriormente indicados se llevaron a cabo mediante el programa gratuito R y el paquete FactoMineR.

\section{Resultados y discusión}

Lo primero a observar en la Figura 1, corresponde a la tasa de crecimiento intercensal de Valdivia en el contexto nacional. Como se aprecia, la ciudad exhibe una baja considerable si se compara tanto a nivel regional como nacional, pese un leve período de alza desde 1952 a 1960. A partir de esta fecha la ciudad presenta un aletargamiento en su dinamismo sostenido en el tiempo.

Tales procesos se deben a diversas causas, pero al menos podrían destacarse 3 . El primero referente al factor tectónico con el terremoto 1960 de 9,5 en la escala de Richter (el sismo con mayor intensidad registrado en el mundo) que afectó gran parte del país, pero tuvo su epicentro en las proximidades de Valdivia, el que fue seguido de un tsunami de proporciones, procesos que destruyeron gran parte de las ciudades y bastas localidades de la costa.

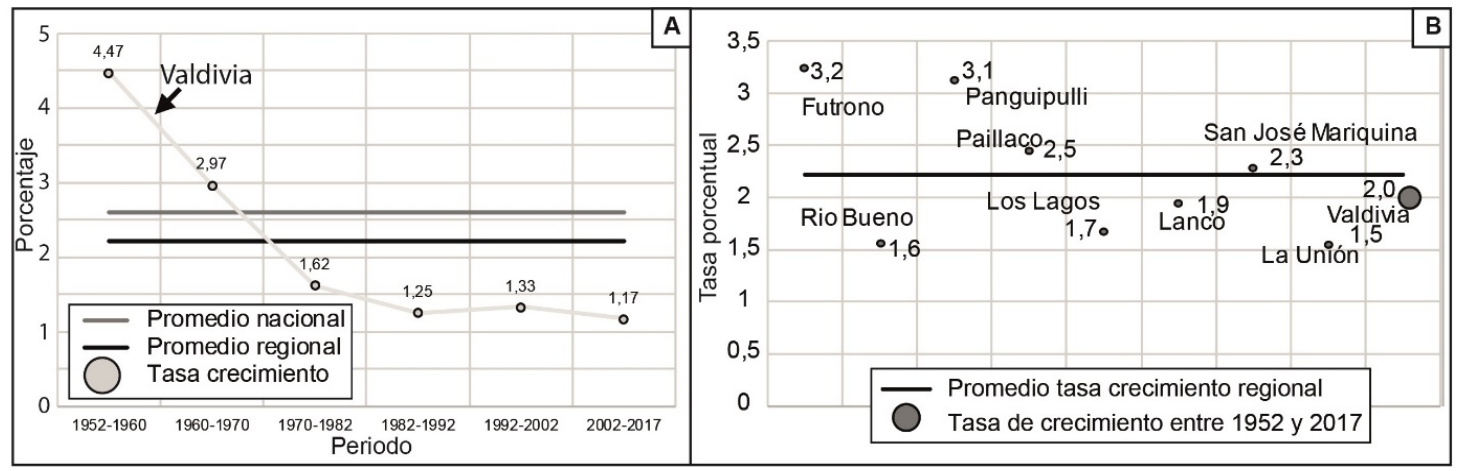

Figura 1 - Tasa crecimiento población ciudad de Valdivia, comparada al promedio regional y nacional (A) y tasas de crecimiento comparada en las ciudades de la Región de Los Ríos entre 1952-2017 (B). Fuente:

Elaboración propia a partir de INE $(1999,2005,2017)$. 
Una segunda causa, se podría atribuir a la pérdida de su rol como capital provincial tras el proceso de regionalización realizado en 1975 durante la dictadura. En efecto, la ciudad quedó privada de una parte relevante de empleos públicos e influencia regional. Tal situación provocó un descontento generalizado en la ciudadanía y que desde el momento de su aplicación trajo consigo reivindicaciones sostenidas, que tuvieron como punto culmine el año 2007, cuando logra ser nuevamente capital regional, pero en esta instancia de la nueva Región de Los Ríos, territorio desprendido de la Región de Los Lagos.

Un tercer elemento que reforzaría lo anteriormente indicado, sería su localización excluida de la principal ruta que atraviesa el país de norte a sur (Ruta 5), lo cual sin duda desconectó a la urbe al no ser un paso obligado por camiones, automovilistas u otros hacia el sur.

Si se analiza a nivel regional, la situación para la ciudad no se modifica sustancialmente (ver Figura 1B). En efecto, las tasas de crecimiento expresan un aletargamiento en comparación a otras ciudades de su espacio regional (Figura 1B). La capital regional durante este período creció de aproximadamente 43.000 habitantes a un total de 143.000 , es decir, sólo 3,3, mientras que, en comparación con otros asentamientos regionales, como por ejemplo Futrono o Panguipulli, ambas han crecido 6 veces su población durante el mismo período de tiempo. Si bien las magnitudes no necesariamente son relevantes, si dan pie a reflexionar en torno a las dinámicas que están produciendo en tales espacios. En efecto, tal situación se ha debido principalmente a la actividad turística, la cual se ha llevado con fuerza en la zona cordillerana de la Región de Los Ríos. Por ejemplo, Panguipulli y Futrono concentran el 12\% y el $9 \%$ respectivamente, de los alojamientos turísticos totales de la región (SERNATUR, 2014). Si bien Valdivia es la primera ciudad en cuestión en términos relativos, las dinámicas recientes de tal actividad estarían impulsando las economías locales.

Otro aspecto que deja de manifiesto tal situación, es la expresada en la Figura 2 que exhibe la evolución en la distribución de la población en los centros poblados en 4 momentos, a partir de la ley rango tamaño. Al observar las curvas de los años 1952, 1970, 2002 y 2017, se puede apreciar como las ciudades de menor tamaño han ido tomando protagonismo de forma notable en el sistema regional, es decir la balanza del equilibrio en la distribución de población estaría siendo desviada hacia las medianas y pequeñas ciudades, mostrando un re equilibrio notable en la población.

Si bien la diferencia entre Valdivia y las ciudades de La Unión (segunda en la jerarquía) y Río Bueno (tercera) ha aumentado sostenidamente en el tiempo. De manera global, las pendientes de las curvas del año 2002 y 2017 tienden a ser considerablemente menos abruptas que los años anteriores, lo que en términos urbanos es bastante positivo para el sistema regional de ciudades. Es decir, las ciudades más pequeñas del sistema han sido bastante dinámicas y esto queda plasmado en los resultados que arroja la curva del último censo de población del año 2017.

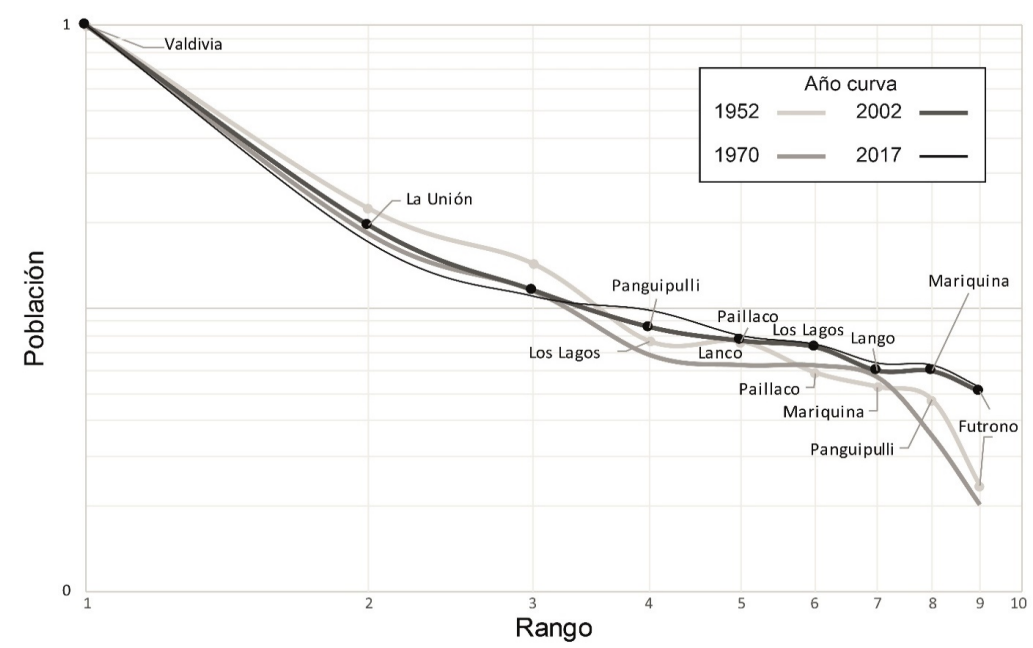

Figura 2 - Evolución de la Población de ciudades regionales a partir de la ley rango tamaño. Fuente: Elaboración propia a partir de INE $(1999,2005,2017)$. 
A raíz de lo expuesto en el análisis, se concluye de manera positiva que la ciudad de Valdivia no sólo no ha logrado polarizar su espacio regional en términos de población, sino que además las diferentes ciudades que componen tal sistema territorial presentan dinámicas positivas de crecimiento y han logrado ser competitivas. Si bien las magnitudes son diferentes, el escenario proyectado sería positivo.

Lo anterior, no quita que la ciudad de Valdivia esté presentando dinámicas urbanas relevantes al interior de su propio espacio urbano, pero a la luz de los primeros datos acá observados, se podrían pensar que son más bien moderadas, lo cual no necesariamente sería negativo.

Por tanto, el siguiente paso fue analizar algunas características de la expansión urbana que han sido evidenciadas en la ciudad. La Tabla 1 -que presenta la variación promedio anual de la expansión en superficie construida de la ciudad- exhibe que tal proceso ha sido más bien constante y sin una expansión explosiva. Por ejemplo, entre 1988 y el 2001 la variación porcentual anual fue de un 1,8 \% mientras que para el 2011 y 2015 fue de 1,9\%. Si bien, se analiza por promedio anual en función de los periodos disponibles (lo que podría sesgar la expansión particular en algún momento), en general no existen ciclos o un momento particular a destacar. Incluso, en los últimos años podría ser más atenuada tal dinámica expansiva, al considerar que durante el periodo 2001-2011 expresó una variación de sólo 1,4\% (ver Tabla 1). Todo lo indicado, no resta que cambios a nivel morfológico puedan ser relevantes y como se verá en las próximas páginas estos resultantes evidentes.

Tabla 1 - Variación porcentual área urbana ciudad Valdivia entre 1988 y 2015

\begin{tabular}{cccc}
\hline & \multicolumn{3}{c}{ Variación Porcentual Áreas } \\
\hline Periodo & $1988-2001$ & $2001-2011$ & $2011-2015$ \\
Variación porcentual anual & $1,8 \%$ & $1,4 \%$ & $1,9 \%$ \\
\hline
\end{tabular}

Fuente: Elaboración propia.

Si se analiza la expansión de la superficie construida, esta se ha desarrollado principalmente hacia el sector sur de la ciudad, y parte norte de Isla Teja y Las Ánimas (ver Figura 3). El crecimiento de la urbe hacia el sur, posiblemente se debe a la conectividad que existe respecto al sector sureste de la ciudad con la Av. Ramón Picarte que luego se convierte en la ruta 206, principal ruta que conecta la capital regional con el sector de Lago Ranco, y que también conecta con la Ruta 5 hacia el sur, llegando a las ciudades de Paillaco, Río Bueno y, posteriormente, la conexión más expedita con la ciudad de Osorno.
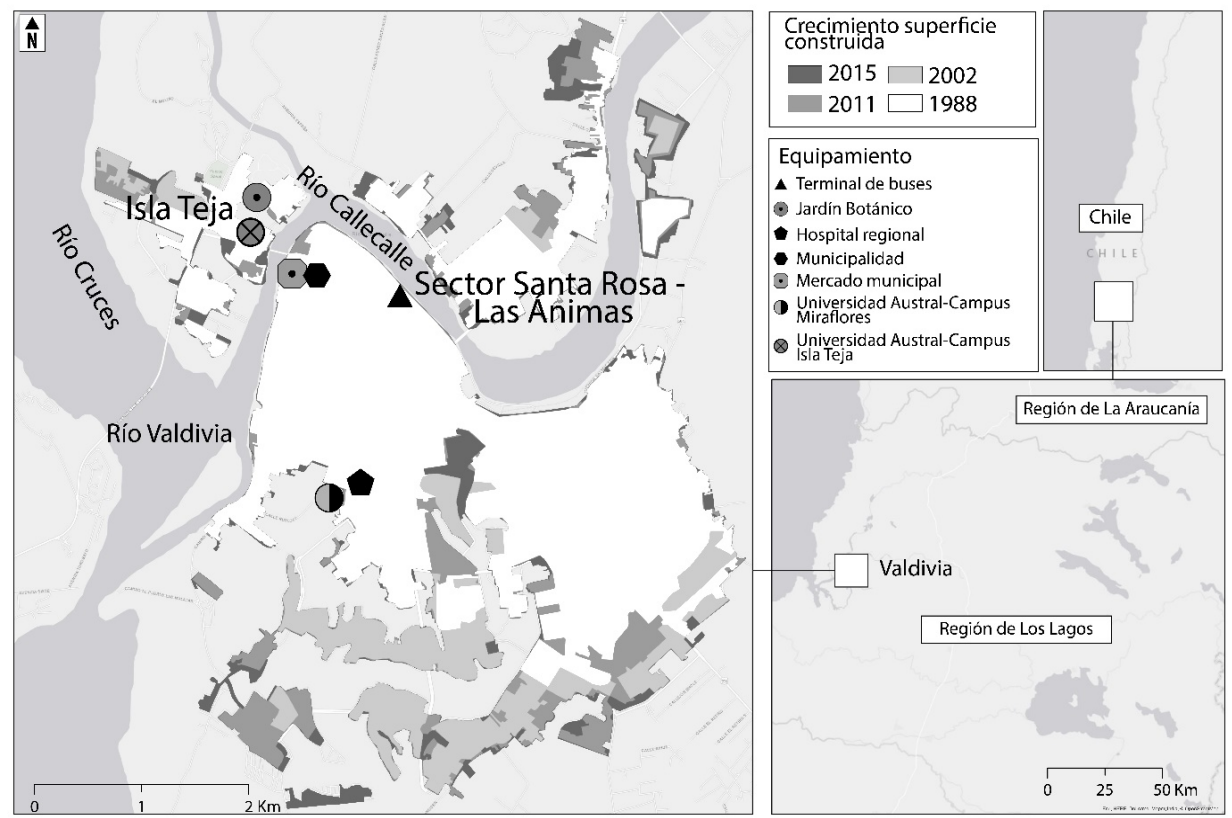

Figura 3 - Variación superficie Construida Ciudad Valdivia (1988-2015), Equipamientos y Contexto regional. Fuente: Elaboración propia. 
No obstante, como ya fue indicado, el fenómeno más interesante a destacar, serían los cambios morfológicos experimentados por la ciudad, los cuales obedecen al proceso de verticalización, expresado en la concentración locacional y densificación en altura (Ramírez et al., 2015).

Para analizar las áreas en que se ha desarrollado la expansión urbana, se elaboró la Figura 4A que presenta desde el año 2002 al 2012 tal información. Se puede apreciar que para el primer año en cuestión, el principal sector que dinamizaba la construcción era el comercio, por consiguiente, se podría entender que durante el período 2001-2011 en términos de la superficie construida (Figura 4A), el crecimiento en el área fue caracterizado principalmente por la función terciaria, seguido por la construcción en tipología de vivienda aislada y otros usos, los cuales se vinculan principalmente al uso residencial pero baja en altura, es decir vivienda unifamiliar. Sin embargo, si observamos el año 2012 (ver Figura 4A), la situación cambió de manera radical. La construcción en altura se dispara y pasa a ser un $100 \%$ en la ciudad de Valdivia respecto a su contexto regional (ver Figura 4A y 4B). Este cambio en el "pattern" de edificabilidades puede explicarse, de acuerdo a la teoría de valores urbanos (Jaramillo, 2003), mediante la acción sincrónica y concurrente de 7 factores de planificación urbana, tales como; (1) aumento en los coeficientes de ocupación de suelo que permite un mayor y mejor aprovechamiento del terreno; (2) incremento en los índices de constructibilidad, lo que se traduce fácticamente en densificación en altura; (3) el interuso vertical y como expresión de mixturas de usos terciarios y residencial; (4) externalidades urbanísticas y grado de consolidación en urbanización dentro del área de estudio; (5) aumento de la demanda solvente de la región, acceso al crédito, capacidad de endeudamiento y fortalecimiento de compra mediante instrumentos financieros; (6) fortalecimiento de las economías de aglomeración regionales; y (7) procesos naturales de segmentación de mercado vinculado a rentas de externalidad regional.

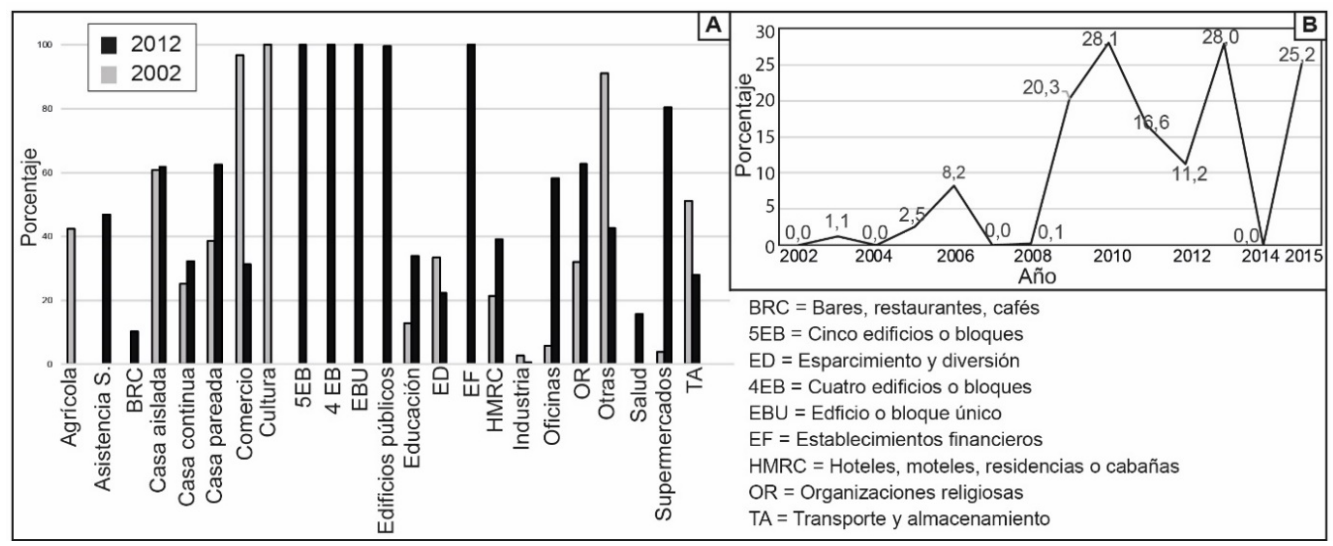

Figura 4 - Permisos edificación en $\mathrm{m}^{2}$, Porcentaje ciudad de Valdivia respecto al total de la Región de Los Ríos, por sectores para años 2002 y 2012 (A) y Número de edificios contruidos en la ciudad de Valdivia respecto al total de permisos residenciales para Valdivia entre 202 y 2015 (B).

Fuente: Elaboración propia a partir de INE (2016).

En efecto, si complementamos tal información con la Figura 4B de Porcentaje de edificios construidos respecto al total de permisos residencial para la ciudad de Valdivia entre 2002 y 2015, desde el año 2009 existe un alza significativa en este tipo de construcciones que pasa a representar un $28 \%$ del total. Situación que se repite para el 2012 y para el año 2015, alcanzando un 25\% del total de los permisos residenciales. Si bien la misma figura muestra algunos ciclos en tal proceso (el año 2014 no se registró algún edificio), es evidente la tendencia al alza y estaríamos frente a un proceso de verticalización relevante.

Otro aspecto a destacar en la Figura 4A, corresponde a los permisos orientados a los edificios públicos, financieros, oficinas y supermercados. Como se observa en la figura, la ciudad de Valdivia concentra gran parte de los permisos en la región, el rol de capital regional otorgado el año 2007 sin duda pudo ser determinante en tal situación. 
Con respecto a la verticalización o densificación en altura residencial, esta se puede caracterizar gracias al apoyo de la Figura 5. Tal cartografía permite apreciar, como la verticalización de la ciudad se concentra principalmente alrededor de la costanera que bordea el Río Valdivia y el Río Callecalle, comprendiendo las calles de General Lagos, Yerbas Buenas y principalmente en la Av. Arturo Prat (ver Figura 5). Con respecto al sector Santa Rosa-Las Ánimas, este se encuentra con la presencia de un tipo de conjuntos habitacionales, que corresponden a la Corporación de la Vivienda creado el año 1974, pero no a edificaciones de tipo altura que se han considerado en el estudio y que se caracterizan por conjuntos que han sido generados por las inmobiliarias orientadas al mercado privado.

En el sector de Isla Teja, la concentración de edificaciones en altura de tipo habitacional se genera en dos sectores: principalmente en el sector sur de esta, con vista hacia el Río Cruces y también con algunas edificaciones hacia el Río Valdivia y, por otra parte, se genera un segundo foco de concentración en el sector noroeste de la isla, con miras únicamente hacia el Río Cruces (ver Figura 5). Además, también cuenta con algunas edificaciones de forma dispersa en el centro de la isla (ver Figura 5).

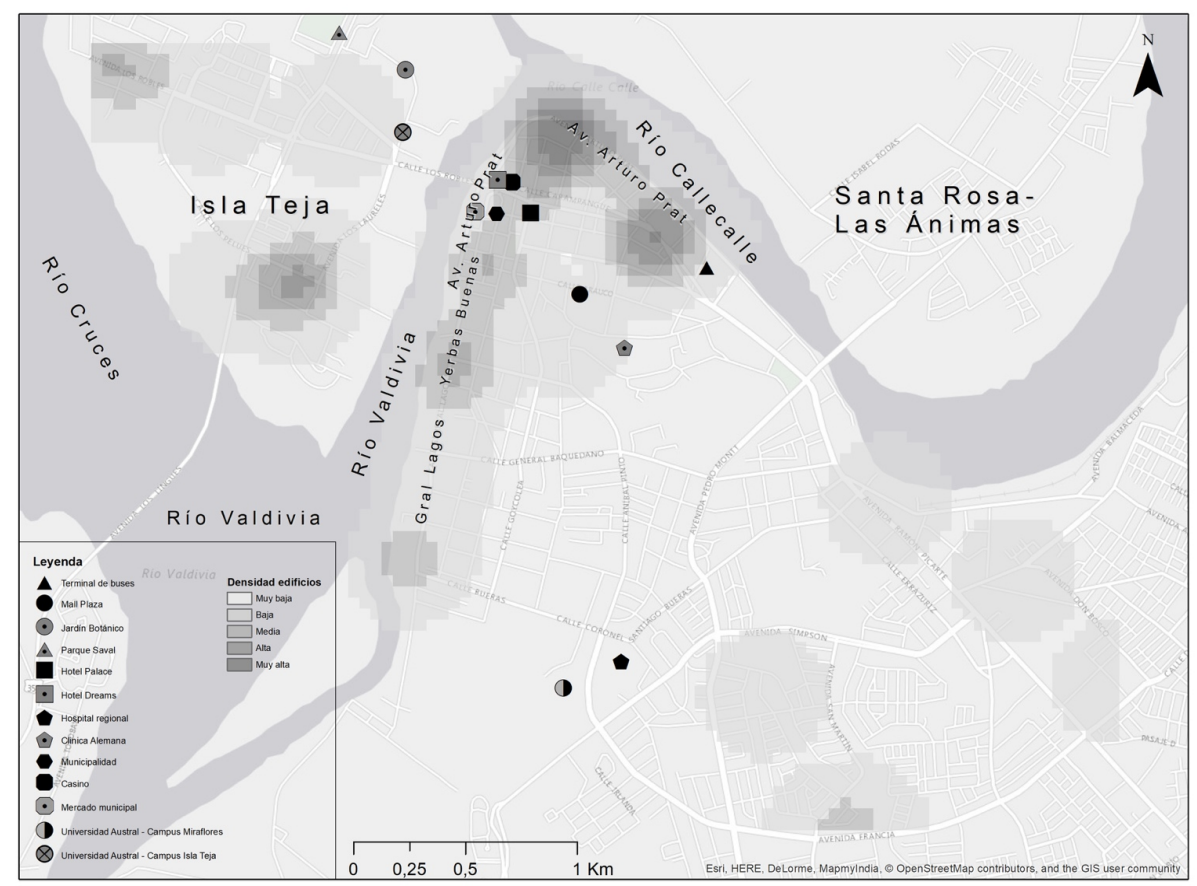

Figura 5 - Densidad de Kernel en Edificación en altura tipo habitacional y Equipamientos, Valdivia. Fuente: Elaboración propia.

Por tanto, se observa que la construcción de edificación en altura de tipo habitacional, se concentra, tanto en el sector de Isla Teja, como en el sector centro de la ciudad, principalmente en puntos en los cuales se aproxima y se visualiza la principal característica hidrográfica de la capital de la Región de Los Ríos, sus cursos fluviales. Lo descrito no es casual, puesto que la ciudad de Valdivia, pretende invertir al año 2017 US\$ 41 millones en el fortalecimiento de la ribera del Río Valdivia y Río Callecalle (Henríquez \& Riquelme, 2017) en tres iniciativas: el Museo de Arte Contemporáneo, en la costanera y en el Centro cívico, esto con el objetivo de contribuir al fortalecimiento de la identidad ribereña de la ciudad de Valdivia.

Lo anterior, sumado a la oferta y equipamientos ya existente principalmente en la costanera, como lo son diferentes hoteles (Palace, Dreams, entre otros), Casino, Mercado Municipal, Municipalidad, entre otros (ver Figura 5), y lo que representaría un mejoramiento en el equipamiento e infraestructura de la costanera planificado para invertir durante el 2017, podrían conllevar a una mayor valorización de ciertas zonas ribereñas en donde las inmobiliarias ya estarían encontrando una oportunidad para invertir.

Así, se buscó relacionar algunos elementos de precios y localización de la verticalización. Por ejemplo, un departamento en el sector noroeste de Isla Teja correspondiente a la esquina de las calles Pintor 
Mauricio Rugendas con Pintor Rodulfo Petersen (ver Figura 6) de $122 \mathrm{~m} 2$, bordea los 6.800 UF7, mientras que en sectores como Av. Arturo Prat es posible encontrar departamentos de 41,4 m2 a 3.523 UF. Los precios de los departamentos van disminuyendo como también sus $\mathrm{m} 2$ a medida que aumenta la distancia al centro, en donde es posible encontrar la mayor parte de los equipamientos (ver Figura 6), por ejemplo, es posible encontrar departamentos en Gral Lagos 950 con un precio de 2.363 UF, con 55 m2.

En sectores céntricos, se puede observar también que, mientras más lejano del Río Valdivia y Callecalle se encuentre el edificio en altura, más disminuye el valor de los departamentos. Por ejemplo, un departamento en Patricio Lynch 930, sector sureste de Valdivia, su precio puede bordear los 1.700 UF con $32,91 \mathrm{~m} 2$, es decir prácticamente 4 veces menos que en los sectores de Isla Teja, calle Pintor M. Rugendas.

La relación entre precio y localización podría explicarse en el concepto de jerarquización de mercado. Considerando que los valores cuantifican una cualificación diferenciada de la estructura urbana (Schovelin \& Roca, 2016). En este sentido, es valor de las externalidades positivas y no el valor unitario o global del suelo el que justifica el patrón de verticalización. Para el caso de Valdivia, son las externalidades, escenográficas, de equipamiento e infraestructura, entendidas como la calidad urbanística del entorno, el instrumento idóneo que permite identificar la estructura jerárquica del mercado urbano (Schovelin \& Roca, 2016). Debido a que la jerarquización distingue y singulariza dentro del valor lo que se debe exclusivamente a la localización espacial de lo que es producto del "pattern" de edificabilidades. Es decir, relaciona el valor de situación urbana en función de atributos. La jerarquización de mercado reconoce, entonces, la profunda relación entre el mercado de suelo y la distribución del producto edificado (Schovelin \& Roca, 2016).

Desde esta perspectiva, podría hipotetizarse que el objeto de estudio estaría sujeto a un proceso de verticalización por rentas de accesibilidad, externalidades urbanísticas y jerarquización social (Schovelin \& Roca, 2016).

Por lo anteriormente indicado, se podría concluir que la tendencia a verticalización urbana de Valdivia estaría fundamentada en relación a tres factores fundamentales; la jerarquización por externalidades paisajísticas, la accesibilidad a equipamiento y los servicios urbanísticos (Ramírez et al., 2015). Estos factores confirmarían en suma que la estructura espacial de la ciudad se basa en la articulación compleja demanda por externalidades, jerarquización social y segmentación de mercado.
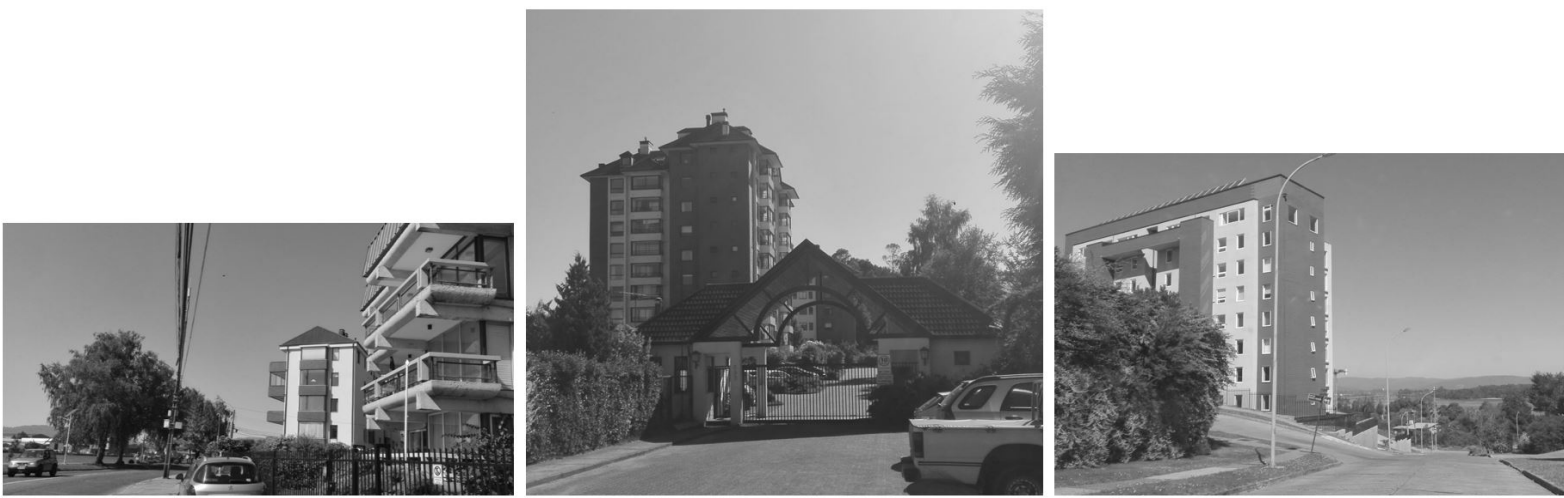

Figura 6 - Ejemplos de Edificios en Av. Arturo Prat, Costanera, Edificio Calle Los Arrayanes \#390, Sector sur, Isla Teja, Edificio Esquina Pintor Mauricio Rugendas con Pintor Rodulfo Petersen, Sector noroeste, Isla Teja. Fuente: Colección propia, tomada el 22 de marzo del 2017.

En este contexto, se ha relatado como existe un incipiente proceso de verticalización y expansión urbana, no obstante, tales las dinámicas no aparecen explosivas y que estén afectando la sustentabilidad de la ciudad. En efecto, una de las razones que podría explicar tal situación, es la dinámica productiva de la ciudad vinculada principalmente al tipo de actividad económica que realiza o posiblemente a que tal

\footnotetext{
7 La UF, Unidad de Fomento, es una unidad financiera reajustable de acuerdo con la inflación (medida según el Índice de Precios al Consumidor o IPC). Es usada en la República de Chile y creada en 1967, siendo su uso original en los préstamos hipotecarios, ya que es una forma de revalorizarlos de acuerdo con las variaciones de la inflación (Valor UF, 2018).
} 
urbe no ha logrado polarizar su espacio regional y por tanto generar fuertes procesos de concentración por atracción de la población, explicada principalmente por una concentración en la oferta de actividades vinculadas al sector servicios más especializados en salud, ocio o educación, por ejemplo.

Lo anterior, toma fuerza al comparar tal situación con lo que ha sucedido en la capital regional de la región vecina, la ciudad de Temuco. En la cual Maturana et al. (2018) evidencian cómo este centro urbano ha tendido a concentrar gran parte de los servicios de la región y anexar funcionalmente una red de centros en torno a ella de aproximadamente 40 kilómetros. Esto, reforzado por profundos cambios en la estructura económica que han presentado algunos territorios de la región y que han ocasionado migraciones relevantes de la zona costera y norte al territorio central de tal región, desarrollando así en Temuco una transformación espectacular en las áreas de servicio y construcción.

Así, se estimó necesario apreciar cómo ha evolucionado el sector del empleo en la ciudad de Valdivia desde 1982 al año 2017, gracias a las ya indicadas bases censales y considerando la importancia de contar con los últimos datos censales que vienen de ser publicados.

En la Figura 7, las primeras observaciones, expresan que durante tal periodo existe una diminución constante del sector agrícola, pesca, silvicultura e industria. Por otro lado, se evidencia un aumento relevante en las áreas del comercio, construcción, hotelería y restaurantes, transporte y comunicaciones, servicios varios (S) y el sector enseñanza. Tales características, en concordancia con lo observado en cuanto a la especialización terciaria que ha sido profundizada en las últimas décadas. Principalmente podrían asociarse al sector enseñanza con la importancia de la Universidad Austral de Chile, una de las más importantes y prestigiosas universidades del país y el sector turismo.

Otro aspecto a destacar, es la variable administración pública, que, si bien presentaba un descenso constante de empleos en tal sector desde 1982 hasta el año 2002, se aprecia un importante repunte al año 2017 atribuible al hecho de haberse convertido en capital regional en el año 2007, lo cual es bastante considerable al observar los datos, en efecto, alcanza prácticamente los mismos niveles del año 1992 (ver Figura 7 variable AD).

Así, a modo de síntesis y a la luz de los datos acá expuestos, Valdivia, en términos funcionales ha tendido a ser poco especializada en un área particular y no es posible observar variaciones o una especialización mayor, lo cual va en línea a otras ciudades en el mundo (como por ejemplo en Francia u otras ciudades de Europa), en cuanto a que mientras mayor sea su cantidad de población tienden a ser menormente especializadas y existe una fuerte relación entre el tamaño y diversas actividades económicas (Bettencourt et al., 2009; Pumain et al., 2009; Pumain \& Saint-Julien, 1996).

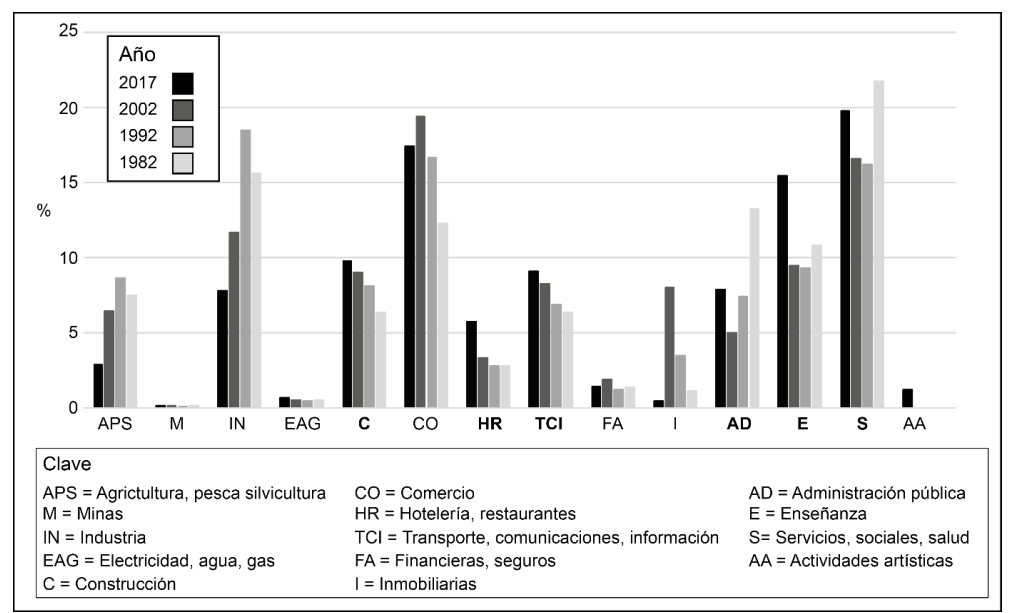

Figura 7 - Evolución del empleo en la ciudad de Valdivia entre 1982 y $2017^{8}$. Fuente: Elaboración propia a partir de INE $(1982,1992,2002,2017)$.

\footnotetext{
${ }^{8}$ Para poder elaborar esta gráfica, se fusionan algunas variables de la clasificación CIIU.
} 
De manera complementaria al análisis expuesto, se llevó a cabo un análisis de componentes principales ACP. En la Figura 8 se aprecia el ACP realizado para los diferentes años. Se presentan 2 gráficos para cada año en los cuales se expresan las cuatro primeras componentes que explican una varianza significativa en cada momento (sobre el $80 \%$ ). Además, sólo se presentan las comunas mejor proyectadas en las diferentes componentes, mismo para las variables (además, que más contribuyen a la formación de la componente), puesto que lo anterior permite una correcta interpretación de tales resultados (Husson et al., 2011).

Cabe destacar -como ya se mencionó en el método- este análisis se realizó de manera regional para observar determinadas particularidades en el conjunto del sistema.

Para el año 1982, las 2 primeras componentes (ver Figura 8), explican prácticamente el 80\% del juego de datos. Se aprecia claramente 4 áreas de especialización. Pesca, Agricultura, empleos vinculados al sector público y de manera general un conjunto de variables vinculadas al sector servicios (componente 1 en su lado positivo) donde la ciudad de Valdivia se constituye en el único centro poblado con tales vínculos y en menor medida La Unión.

Para el año 1992, la situación aparece similar. Valdivia vinculada al sector servicio, misma que La Unión, aunque emerge también Lanco. Lo que destaca en este periodo, es la perdida de relevancia en empleos vinculados al sector públicos y resta solamente el área de la pesca y agrícola vinculada a más comunas de la región.

Para el año 2002, la situación aparece similar, salvo algunos elementos a destacar en las componentes 3 y 4 , donde es posible ver una importancia relativa del sector construcción, industrial y vinculados al turismo como hotelería y restauración.

Finalmente, para el año 2017, Valdivia refuerza su posición como la ciudad mas relevante en las diferentes variables vinculadas al sector terciario. Panguipulli con una importante vocación turística y las ciudades de Máfil y Río Bueno orientadas al sector agrícola.

Por lo visto, en términos generales, la especialización en cada uno de los territorios sigue bastante estable y Valdivia orientada a los servicios de manera más destacada y particular. No obstante, futuros análisis desde un enfoque regional, podrían evidenciar que las magnitudes de la especialización de otras comunas, podrían presentar algunos matices. 


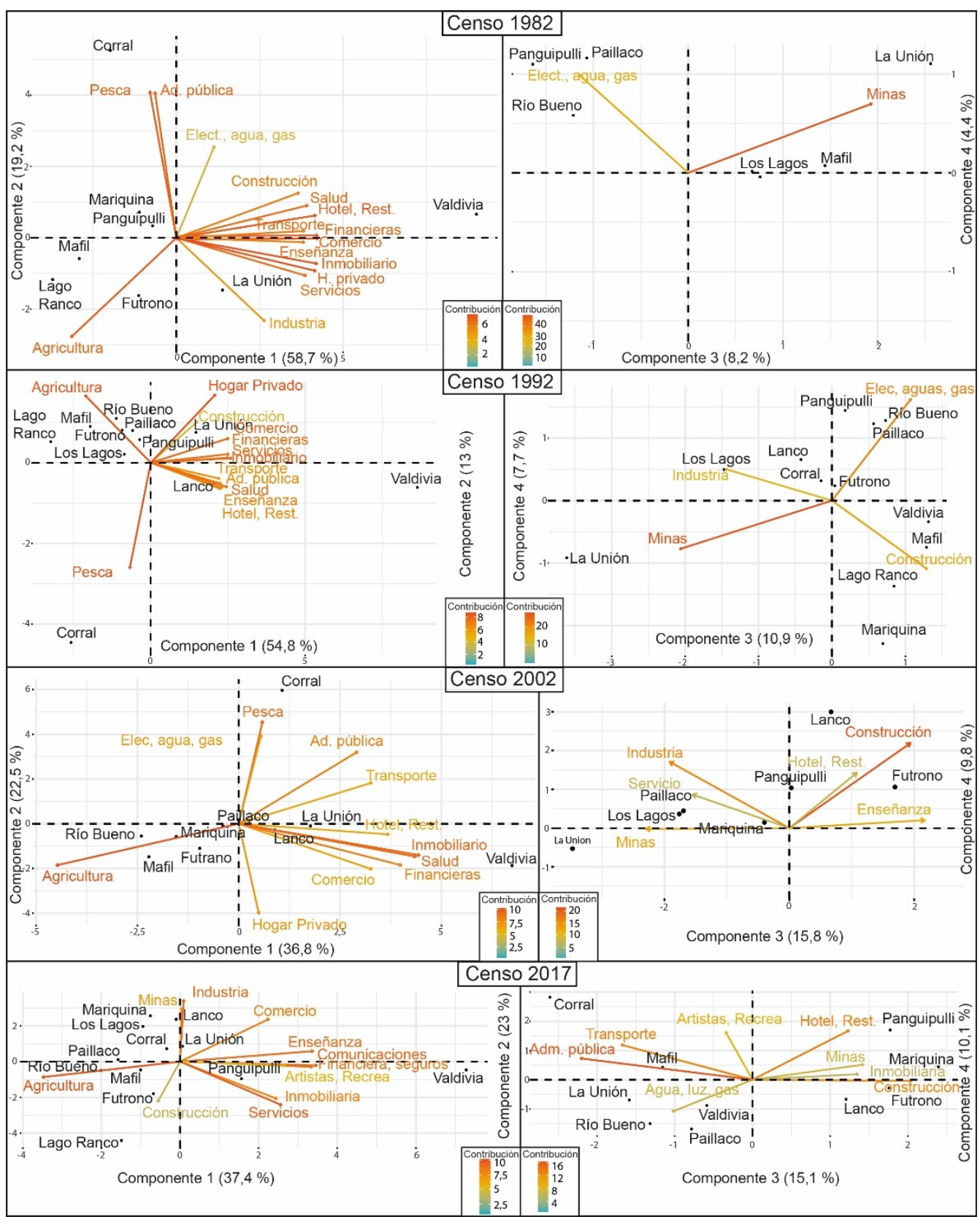

Figura 8 - Análisis de componentes principales de las actividades económicas de los habitantes en las ciudades de la Región de Los Ríos`. Fuente: Elaborac£ión propia a partir de INE (1982, 1992, 2002, 2017).

\section{Conclusión}

La urbanización plantea desafíos en términos a cómo van a crecer y articularse las ciudades.

En este caso particular para la ciudad de Valdivia, mediante la comprobación empírica de los factores estudiados se han demostrado dinámicas urbanas relevantes, pero que, pese a su rol como capital regional, no se ha desencadenado en la actualidad un proceso de verticalización veloz que esté modificando drásticamente la ciudad.

Lo anterior podría deberse a varias causas, la primera más bien en términos de su localización. Al distar la ciudad de centros urbanos próximos, esto ha impedido que Valdivia "anexe" algunos sub centros

\footnotetext{
${ }^{9}$ Algunas variables de la clasificación CIIU para el año 2017 fueron agrupadas para su mejor presentación.
} 
a su espacio y más bien se ha desencadenado un crecimiento limitado a sus bordes sin una configuración policéntrica, donde se exhiban movimientos pendulares de relevancia.

Un segundo aspecto, corresponde que a pesar de concentrar buena parte de los servicios que dispone la región y las instituciones desconcentradas del Estado, tal situación no ha provocado una polarización de su espacio regional, obedeciendo posiblemente lo anterior, es decir, que algunas comunas de la región han logrado mediante la actividad turística generar un bienestar que no incentiva procesos migratorios de relevancia.

Una tercera razón es que los fenómenos de crecimiento urbano funcionan competitivamente y se estructuran alrededor de la optimización de las localizaciones, entendiendo la optimización como la maximización de las utilidades conseguidas a través la concentración de externalidades físicas. Estas externalidades lejos de ser estáticas comportan dinamismo en su evolución temporal, de forma tal que los procesos de crecimiento conducirán, por jerarquización de mercado, a la maximización en uso vertical del suelo. En este sentido, los antecedentes recogidos durante esta investigación sugieren que existe un creciente proceso de urbanización por densificación en altura, que ha sido el resultado de la aplicación de iniciativas públicas de transformación espacial. Sin embargo, las decisiones de planificación y gestión territorial deben apuntar a una política de control frente al incipiente fenómeno de metropolización de Valdivia.

Si bien estas conclusiones deberían ser profundizadas, es interesante constatar que la matriz económica la ciudad impediría un proceso de expansión explosiva cómo si se ha visto en otras ciudades producto del vínculo a producción de materias primas, como por ejemplo Puerto Montt, capital regional de región contigua o administradora de "pobreza" como lo sería Temuco al polarizar a la Región de La Araucanía que es actualmente la que presenta las tasas de pobreza más altas del país.

Así, tal proceso que afecta a la ciudad de Valdivia podría verse de manera positiva al no existir una polizariación del espacio y un crecimiento urbano contenido, pero existen luces de atención en cuanto a la verticalización de la ciudad, que debe ir acompañada de un instrumento de planificación actualizado. En este caso, el Plano Regulador Comunal data del año 1988 y la nueva versión no ha logrado dar a luz, por tanto, se debe pensar en tales aspectos para entregarle un mejor apronte a la ciudad, frente a los cambios que puedan venir.

\section{Referencias}

Aguayo, M., Pauchard, A., Azócar, G., \& Parra, O. (2009). Cambio del uso del suelo en el centro sur de Chile a fines del siglo XX: entendiendo la dinámica espacial y temporal del paisaje. Revista Chilena de Historia Natural, (82), 361-374. http://dx.doi.org/10.4067/S0716-078X2009000300004.

Barton, J. R., Jordán Fuchs, R., León, A., Mabel, S., \& Solis, M. (2007). ¿Cuán sustentable es la Región Metropolitana de Santiago?: metodologías de evaluación de la sustentabilidad. Santiago: Comisión Económica para América Latina y el Caribe (CEPAL).

Bellet, C., Melazzo, E., Beltrão Sposito, M. E., \& Llop, J. (Eds.). (2015). Urbanización, producción y consumo en ciudades intermedias. Lleida: Edicions de la Universitat de Lleida.

Bettencourt, L., Lobo, J., \& West, G. (2009). The self similarity of human social organization and dynamics in cities. In D. Lane, S. Ernst van der Leeuw, D. Pumain, \& G. West (Eds.), Complexity perspectives in innovation and social change (pp. 221-236). Dordrecht: Springer. http://dx.doi.org/10.1007/978-1-4020-9663-1_8.

Bitoun, J., Miranda, L., \& Moura, R. (2017). Cidades médias no Brasil: heterogeneidade, diversidade, e inserção nos espaços rurais brasileiros. In F. Maturana, M. E. Beltrão Sposito, C. Bellet, F. Arenas, \& C. Henríquez, (Eds.), Sistemas urbanos y ciudades medias en Iberoamérica (pp. 44-79, Serie GeoLibros). Santiago: Instituto de Geografía, Pontificia Universidad Católica de Chile.

Borja, J. (2005). La ciudad en la globalización. In C. Arce, E. Cabrero, \& A. Ziccardi (Eds.), Ciudades del siglo XXI: ¿competitividad o cooperación? (pp. 65-101). México: Centro de Investigación y Docencia Económica (CIDE). 
Borsdorf, A. (2000). El desarrollo urbano de Valdivia: estudio de caso en una ciudad mediana. Espacio y Desarrollo, (12), 45-81.

Borsdorf, A., Sanchez, R., \& Marchant, C. (2009). Las ciudades intermedias aletargadas del sistema urbano chileno y la oportunidad de un desarrollo sustentable: el caso de la ciudad de Valdivia. In C. Bellet, \& M. Beltrão (Eds.), Las ciudades medias o intermedias en un mundo globalizado (pp. 365-388). Lleida: Edicions de la Universitat de Lleida.

Castells, M. (1995). La ciudad informacional: tecnologías de la información, reestructuración económica y el proceso urbano-regional. España: Alianza Editorial.

Harvey, D. (2013). Ciudades rebeldes: del derecho de la ciudad a la revolución urbana. Madrid: Ediciones Akal.

Henríquez, S., \& Riquelme, O. (2017). Ciudades fluviales destinan US\$124 millones en obras para volcarse a sus costaneras. Chile: El Mercurio. Recuperado el 5 de julio del 2018, de

http://impresa.elmercurio.com/Pages/NewsDetail.aspx?dt=2017-03-05\&dtB=05-03-

2017\%200:00:00\&PaginaId=18\&bodyid $=3$

Hidalgo, R., Mattos, C., \& Arenas, F. (2009). Chile: del país urbano al país metropolitano (Serie GEOlibros). Santiago: Pontificia Universidad Católica de Chile.

Husson, F. Lê, S. \& Pagès, J. (2011). Exploratory multivariate analysis by example using R. Boca Raton: Taylor \& Francis Group.

Instituto Nacional de Estadística - INE. (1982). Base censal REDATAM. Santiago: Gobierno de Chile.

Instituto Nacional de Estadística - INE. (1992). Base censal REDATAM. Santiago: Gobierno de Chile.

Instituto Nacional de Estadística - INE. (1999). Población de los centros poblados de Chile: 1875-1992. Santiago:

Gobierno de Chile.

Instituto Nacional de Estadística - INE. (2002). Base censal REDATAM. Santiago: Gobierno de Chile.

Instituto Nacional de Estadística - INE. (2005). Chile: ciudades, pueblos, aldeas y caseríos. Santiago: Gobierno de Chile.

Instituto Nacional de Estadística - INE. (2016). Permisos de edificación. Santiago: Gobierno de Chile. Recuperado el 5 de marzo de 2018, de http://www.ine.cl/estadisticas/economicas/construccion/edificaci\%C3\%B3n-superficieautorizada

Instituto Nacional de Estadística - INE. (2017). Censo nacional de población. Santiago: Gobierno de Chile. Recuperado el 27 de junio del 2018, de http://www.censo2017.cl/

Jaramillo, S. (2003). Los fundamentos económicos de la participación en plusvalías. Bogotá: CIDE Universidad de los Andes, Lincoln Institute of Land Policy.

Lan, D., \& Migueltorena, A. (2017). Aportes para el estudio de ciudades intermedias de Argentina. In F. Maturana, M. E. Beltrão Sposito, C. Bellet, F. Arenas, \& Henríquez, C. (Eds.), Sistemas urbanos y ciudades medias en Iberoamérica (pp. 16-43, Serie GeoLibros). Santiago: Instituto de Geografía, Pontificia Universidad Católica de Chile.

Marengo, C., \& Monayar, V. (2012). Crecimiento urbano e informalidad residencial: el caso Nuestro Hogar III en la periferia de Córdoba, Argentina. Cuaderno Urbano, 13(13), 7-25.

Mattos, C. A. (2010). Globalización y metamorfosis metropolitana en América Latina: de la ciudad a lo urbano generalizado. Revista de Geografía Norte Grande, (47), 81-104.

Maturana, F., \& Rojas, A. (Eds.). (2015). Ciudades intermedias en Chile: territorios olvidados. Santiago: Ril Editores.

Maturana, F., Beltrão Sposito, M. E., Bellet, C., Arenas, F., \& Henríquez, C. (Eds.). (2017). Sistemas urbanos y ciudades medias en Iberoamérica (Serie GeoLibros). Santiago: Instituto de Geografía, Pontificia Universidad Católica de Chile. 
Maturana, F., Rojas, A., \& Salas, R. (2018). Dinámicas espaciales y transición hacia la articulación de espacios metropolitanos: el caso de Temuco y su hinterland, Chile. Cuadernos Geográficos de la Universidad de Granada, 57, 132-154.

Montero, L., \& García, J. (2017). Panorama multidimensional del desarrollo urbano en América Latina y el Caribe. Santiago: Comisión Económica para América Latina y el Caribe (CEPAL).

Montoya, C. (2012). Destrucción creativa. Revista Ciencias Estratégicas, 20(28), 213-216.

Organización de las Naciones Unidas - ONU. (2016). World cities report 2016: urbanization and development emerging futures. Nairobi: United Nations Human Settlements Programme (UN-Habitat).

Pumain, D., \& Saint-Julien, T. (1996). Evolution des spécialisations urbaines et cycles d'innovation. In D. Pumain, \& F. Godard (Eds.), Données urbaines (pp. 247-255). Paris: Anthropos-Economica.

Pumain, D., \& Saint-Julien, T. (2001). Análisis espacial: las interacciones. Concepción: Universidad de Concepción, Pontificia Universidad Católica de Chile.

Pumain, D., Paulus, F., \& Vacchiani-Marcuzzo, C. (2009). Innovation cycles and urban dynamics. In D. Lane, S. Ernst van der Leeuw, D. Pumain, \& G. West (Eds.), Complexity perspectives in innovation and social change (pp. 237-260). Netherlands: Springer. http://dx.doi.org/10.1007/978-1-4020-9663-1_9.

Ramírez, F. (2014). El experticismo desnaturalizado del planificador: ¿un urbanismo participativo o espacio para mercaderes? Revista Trilogía, 24, 51-59.

Ramírez, F., Valencia, M., Olguín, R., Dentice, A., Gajardo, L., \& Mac-Lean, G. (2015). Perspectivas teórico: académica del Desarrollo Urbano: el desarrollo es ante todo un proceso endógeno. In F. Ramírez (Ed.), Neoliberalismo y sustentabilidad ciudadana (pp. 75-82). Santiago: Editorial Quimantú.

Santiago, C. M., Raggi, J. P. F., \& Erices, L. V. (2016). Urban growth trends in midsize Chilean cities: the case of Temuco. urbe. Revista Brasileira de Gestão Urbana, 8(3), 375-389. http://dx.doi.org/10.1590/21753369.008.003.A007.

Santos, M. (2000). La naturaleza del espacio: técnica y tiempo: razón y emoción. Barcelona: Ariel.

Sapir, J. (2004). Economistas contra la democracia: los intereses inconfesables de los expertos de la economía. Barcelona: Ediciones B.S.A.

Schovelin, R., \& Roca, J. (2016). Un modelo para seleccionar atributos de un edificio residencial que maximiza el precio de venta. Obras y Proyectos, 19(19), 61-72. http://dx.doi.org/10.4067/S0718-28132016000100005.

Servicio Nacional de Turismo - SERNATUR. (2014). Estadísticas. Chile: SERNATUR. Recuperado el 5 de marzo de 2018, de http://www.subturismo.gob.cl/documentos/estadisticas/

Smith, N. (1996). The new urban frontier: gentrification and the revanchist city. London: Routledge.

Smith, N. (2005). El redimensionamiento de las ciudades: la globalización y el urbanismo neoliberal. In D. Harvey, \& N. Smith (Eds.), Capital financiero, propiedad inmobiliaria y cultura (pp. 59-78). Barcelona: Universitat Autònoma de Barcelona.

U.S. Geological Survey - USGS. (2018). Recuperado el 5 de marzo de 2018, de https://ers.cr.usgs.gov/

Valor UF. (2018). Recuperado el 5 de marzo de 2018, de http://valoruf.cl/que_es_la_uf.html

Editor: Fábio Duarte

Recibido: Jul. 25, 2018

Aprobado: Oct. 13, 2018 